\title{
The effect of external factors on dielectric permittivity of Rochelle salt: humidity, annealing, stresses, electric field
}

\author{
A.G.Slivka ${ }^{1}$, V.M.Kedyulich ${ }^{1}$, R.R.Levitskii ${ }^{2}$, A.P.Moina ${ }^{2}$, \\ M.O.Romanyuk ${ }^{3}$, A.M.Guivan ${ }^{1}$ \\ 1 Uzhgorod National University, \\ 54 Voloshin Street, 88000 Uzhgorod, Ukraine \\ 2 Institute for Condensed Matter Physics, \\ 1 Svientsitskii Street, 79011 Lviv, Ukraine \\ 3 Ivan Franko Lviv National University, \\ 8 Kyryla and Mefodiya Street, 79005 Lviv, Ukraine
}

Received May 18, 2005

The effect of external factors, such as dessicating/wetting, thermal annealing, uniaxial and hydrostatic pressure, on the dielectric permittivity of Rochelle salt crystals is investigated. The obtained results are compared with the available literature data and analyzed within the phenomenological Landau approach. A significant effect of the internal polar point defects in crystals and storage conditions on the dielectric permittivity is shown.

Key words: Rochelle salt, pressure, electric field, defects, annealing, humidity

PACS: $77.80 . B h, 77.22 . \mathrm{Ch}$

\section{Introduction}

A useful information about ferroelectric crystals can be obtained by exploring their behavior under the influence of various external factors, such as high pressure or electric field. For hydrogen bonded crystals the external pressures are the only way to continuously vary geometric parameters of bonds, to break their equivalence, etc, which permits to investigate the role of hydrogen bonds, their parameters and symmetry the phase transition and dielectric response of the crystals. Many ferroelectrics are piezoelectric in the paraelectric phase; application of shear stresses and the conjugate electric fields allows one to explore the role of piezoelectric interactions in the phase transitions and in the formation of physical characteristics of the crystals. 
These possibilities were fully used for the investigation of the $\mathrm{KH}_{2} \mathrm{PO}_{4}$ family crystals. Theoretical description of pressure and field effects in these crystals is usually performed within the proton ordering model (see e.g. [1-6] and references therein); a quantitative agreement with the experiment is obtained. It was shown, in particular, that pressures of different symmetries produce qualitatively different changes in the phase transition: lower its temperature down (hydrostatic), raise it up and smear out the transition (shear stress $\sigma_{6}$ ), induce a new phase of monoclinic symmetry (as $\left.\sigma_{1}-\sigma_{2}\right)$.

In contrast to the $\mathrm{KH}_{2} \mathrm{PO}_{4}$ family crystals, the data concerning the influence of external factors on Rochelle salt are less extensive. The data, in particular, are available on hydrostatic pressure [7-9] and electric field [10-12] effects on the elastic constant and dielectric permittivity of the crystals. Uniaxial stress effects on the phase transitions in Rochelle salt were explored in [13] from the measurements of thermoelastic effect. Theoretically the effect of the shear stress $\sigma_{4}$ was studied in [14] within the modified Mitsui model.

Usually, physical characteristics of ferroelectric crystals in the vicinity of phase transitions (especially of the second order ones) are affected by crystal defects and internal bias electric fields and mechanical stresses, which act as the external ones. The role of such factors as crystal defectness and the processes in the sample prehistory that may affect the physical properties of the crystals via relaxation of the defects such as thermal annealing, previous action of electromagnetic fields and mechanical stresses should be explored. High pressure and electric field studies make it possible to explore the intrinsic field and pressure dependences of the crystal characteristics, reveal the internal bias fields and stresses, and study the residual effects of the internal defects.

For Rochelle salt, whose efflorescence (loss of crystallization water) and deliquescence (uptake of water) are well known, such a factor as storage air humidity is important and should be monitored during measurements. For instance, a significant dispersion of experimental data for the dielectric permittivity of Rochelle salt (see the systematization in [15]), which exceeds the measurement error, takes place. Apparently, the dispersion is due to different internal states of the samples uncontrolled during measurements.

In the present work, the effect of the above mentioned external factors (pressure, electric field, humidity, thermal annealing) on the dielectric permittivity of Rochelle salt crystals in the vicinity of the structural phase transitions are explored.

\section{Experimental setup}

Dielectric permittivity $\varepsilon_{11}$ of the crystals at $1 \mathrm{kHz}$ was determined by measuring the capacity of the samples by means of an a.c. bridge. Measurement error was $0.2 \div 0.4 \%$. Samples were prepared in the form of parallellepipeds, with the faces perpendicular to the crystallographic axes of the orthorhombic (paraelectric) unit cell. Silver paste and copper wires, $0.08 \div 0.12 \mathrm{~mm}$ diameter, were used as electric contacts. After partial drying of the paste, the contacts were covered by an alcohol 
solution of a glue with addition of silver paste. This method provided a necessary mechanical stability of the contacts and made a free deformation of the crystals possible.

A uniaxial pressure was created by a spring dynamometer and was transmitted to samples via a punch with floating heads, thus securing a uniform pressure even at possibly non-parallel faces of the sample. The pressure was set with an accuracy of $\pm 5 \%$. Temperature was measured using a copper-constantan thermocouple with an accuracy \pm 0.1 . Samples with the thermocouple were covered with silicone oil, in order to enhance heat transmission and prevent direct contact with the air.

\section{Model approach}

Theoretical description of the physical properties of Rochelle salt is usually performed within a two-sublattice Ising model with asymmetric double well potential (Mitsui model). Hereinafter we present the expression for the dielectric permittivity of Rochelle salt obtained within the modified Mitsui model with taking into account the piezoelectric coupling [15] with the Hamiltonian

$$
\begin{aligned}
\widehat{H}=\frac{N}{2} v c_{44}^{E 0} \varepsilon_{4}^{2}-N v e_{14}^{0} \varepsilon_{4} E_{1}-\frac{N}{2} v \chi_{11}^{0} E_{1}^{2}-\frac{1}{2} \sum_{q q^{\prime}} \sum_{f f^{\prime}=1}^{2} R_{q q^{\prime}}\left(f f^{\prime}\right) \frac{\sigma_{q f}}{2} \frac{\sigma_{q^{\prime} f^{\prime}}}{2} \\
-\Delta \sum_{q}\left(\frac{\sigma_{q_{1}}}{2}-\frac{\sigma_{q_{2}}}{2}\right)-\left(\mu_{1} E_{1}-2 \psi_{4} \varepsilon_{4}\right) \sum_{q} \sum_{f=1}^{2} \frac{\sigma_{q f}}{2} .
\end{aligned}
$$

Three first terms in (3.1) correspond to a "seed" energy of the crystal lattice which forms the asymmetric double-well potential for pseudospins. $R_{q q^{\prime}}(11)=R_{q q^{\prime}}(22)=$ $J_{q q^{\prime}}$ and $R_{q q^{\prime}}(12)=R_{q q^{\prime}}(21)=K_{q q^{\prime}}$ are constants of interaction between pseudospins belonging to the same and to different sublattices, respectively. The parameter $\Delta$ describes the asymmetry of the double well potential; $\mu_{1}$ is the effective dipole moment. The last term is the internal field created by the piezoelectric coupling with the shear strain $\varepsilon_{4}$; the quantity $\psi_{4}$ describing this coupling is treated as a model parameter.

Within a mean field approximation the static dielectric permittivity of a free crystal was obtained in the form [15]

$$
\chi_{11}^{\sigma}=\chi_{11}^{\sigma 0}+\frac{\beta\left(\mu_{1}^{\prime}\right)^{2}}{2 v} F_{2} .
$$

The following notations are used

$$
\begin{gathered}
F_{2}=\frac{\varphi_{3}}{\varphi_{2}-\Lambda \varphi_{3}}, \\
\varphi_{2}=1-\frac{\beta J}{2} \lambda_{1}-\beta^{2} \frac{K^{2}-J^{2}}{16}\left(\lambda_{1}^{2}-\lambda_{2}^{2}\right), \quad \varphi_{3}=\lambda_{1}+\beta \frac{K-J}{4}\left(\lambda_{1}^{2}-\lambda_{2}^{2}\right), \quad \Lambda=\frac{2 \beta \psi_{4}^{2}}{v c_{44}^{E 0},} \\
\lambda_{1}=1-\xi^{2}-\sigma^{2}, \quad \lambda_{2}=2 \xi \sigma, \\
\chi_{11}^{\sigma 0}=\chi_{11}^{\varepsilon 0}+e_{14}^{0} d_{14}^{0}, \quad \mu_{1}^{\prime}=\mu_{1}-2 \psi_{4} d_{14}^{0}, \quad d_{14}^{0}=\frac{e_{14}^{0}}{c_{44}^{E 0}},
\end{gathered}
$$


$\xi, \sigma$ are the parameters of ferroelectric and antiferroelectric ordering.

Values of the model parameters providing the best fit to the permittivity are given in table 1 .

Table 1. Model parameters for Rochelle salt [15].

\begin{tabular}{ccccccc}
\hline$J / k_{\mathrm{B}}$ & $K / k_{\mathrm{B}}$ & $\Delta / k_{\mathrm{B}}$ & $\psi_{4} / k_{\mathrm{B}}$ & $\begin{array}{c}c_{44}^{E 0} \\
\mathrm{Kyn} / \mathrm{cm}^{2}\end{array}$ & $\begin{array}{c}d_{14}^{0} \\
\mathrm{esu} / \mathrm{dyn}\end{array}$ & $\chi_{11}^{\sigma 0}$ \\
\hline 797.36 & 1468.83 & 737.33 & -760 & $12.8 \cdot 10^{10}$ & $1.9 \cdot 10^{-8}$ & 0.363 \\
\hline
\end{tabular}

$v=0.5219[1+0.00013(T-190)] \cdot 10^{-21} \mathrm{~cm}^{3}, \quad \mu_{1}=[2.52+0.0066(297-T)] \cdot 10^{-18} \mathrm{esu} \mathrm{cm}$.

\section{The effect of sample prehistory}

\subsection{Humidity}

In [16] it was found that crystals of Rochelle salt at $25^{\circ}$ and at relative humidity below $40 \%$ lose the crystallization water, whereas at relative humidity above $85 \%$ they absorb water from the air. Significant changes of the piezoelectric properties of Rochelle salt were observed in the samples kept in the air with a high concentration of ethanol vapor [17]. An essential effect of humidity on the relaxation times of the dielectric permittivity of Rochelle salt was also observed in [18] and attributed to the concentration changes of water vacancies in the lattice.

Experimental data for susceptibility of Rochelle salt (see figure 1) are strongly dispersed, even in the paraelectric phases. This cannot be accounted for by the differences in the measurement regimes. Thus it was interesting to explore the temperature dependences of Rochelle salt crystals with different water content in order to verify whether this dispersion can be attributed, at least partially, to it.
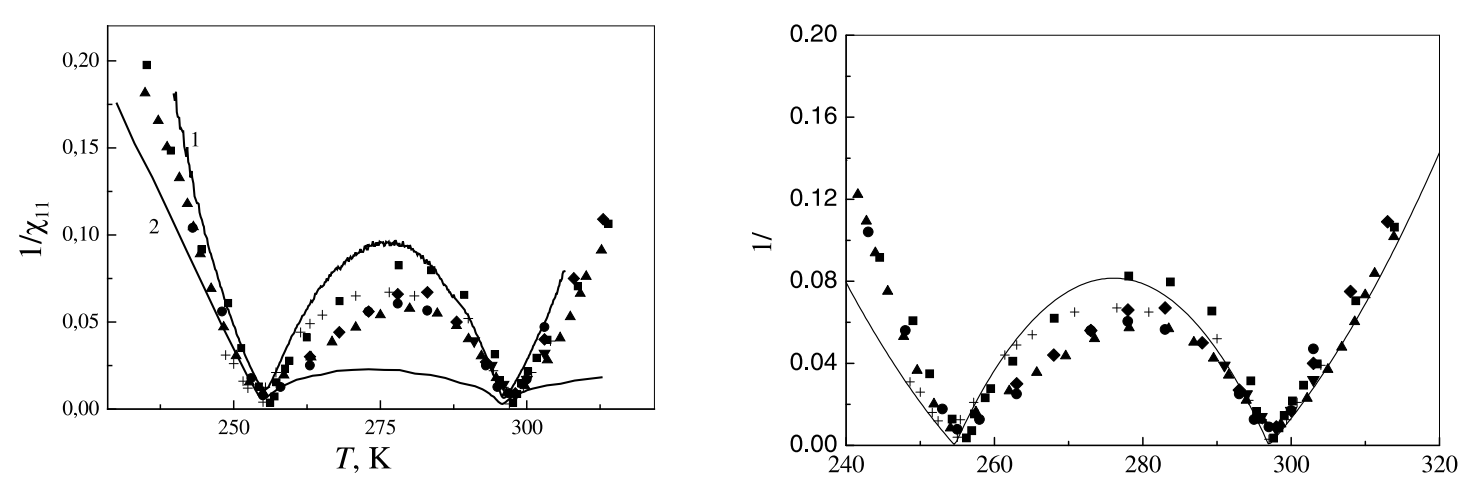

Figure 1. Temperature dependences of inverse susceptibility of Rochelle salt. Left: solid lines - experimental data of this work, obtained for dessicated (1) and wet (2) samples. Right: solid line - theoretical curve, calculated with equation (3.2). $\mathbf{\square}-[19] ; \boldsymbol{\Lambda}-[20] ; \bullet-[21] ; \bullet-[22] ; \boldsymbol{\nabla}-[23] ;+-[24]$. 
The obtained results are presented in figure 1 (left). Along with the literature data, here we show the temperature dependences of the inverse dielectric susceptibility $\chi_{11}^{-1}$ (solid curves 1 and 2), obtained in this work for the same sample with different water content. The curve 1 was obtained for a sample, kept for a long time (2-3 days) at room temperature in a closed volume, filled with a dessicator (silicagel). The curve 2 corresponds to the same sample, kept for 10 hours in the air with relative humidity $\sim 90 \%$. As one can see, keeping the sample in a wet air increases the dielectric susceptibility in the entire temperature range studied. The changes are particularly prominent in the middle of the ferroelectric phase $T \sim 275 \mathrm{~K}$ and in the high-temperature paraelectric phase.

Comparison of the obtained results with literature data shows that the dispersion in the values of the susceptibility can be caused by the different water content in the samples used in different experiments. It should be also noted that for the wet sample (curve 2, figure 1), a linear temperature dependence of the inverse susceptibility is observed with the Curie-Weiss constant $C_{\mathrm{W}}=1.95 \cdot 10^{3} \mathrm{~K}$. For the dessicated sample, this dependence is non-linear in both paraelectric phases.

Comparison of literature experimental data with the theoretical curve ([15] and equation (3.2)), is given in figure 1 (right). Theoretical values of permittivity are adjusted by the value of the effective dipole moment $\mu_{1}$. In [15] $\mu_{1}$ was chosen such that the best agreement with the data of [23] as well as of the dynamic microwave permittivity should be obtained. However, we failed to get an adequate agreement with the experiment for susceptibility in the low-temperature paraelectric phase [15].

\subsection{Thermal annealing}

Figure 2 illustrates the temperature dependences of dielectric permittivity of Rochelle salt near the upper Curie point for samples annealed at $308 \mathrm{~K}$. On increasing the annealing time, the value of the dielectric permittivity at the transition point increases, and the maximum temperature decreases. Such changes are ap-

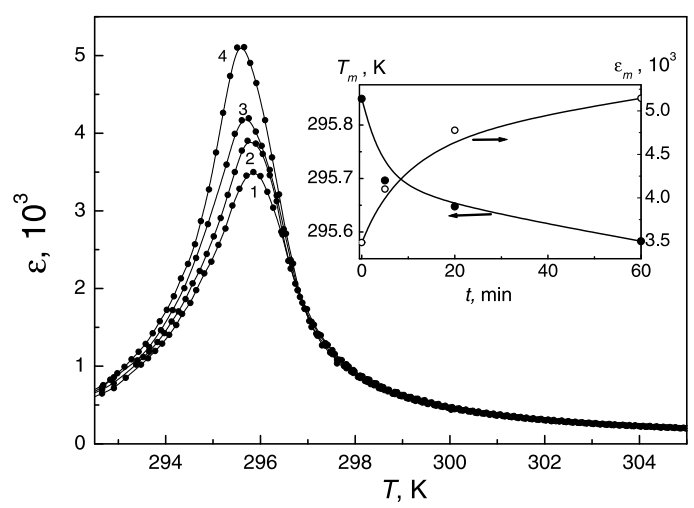

Figure 2. Temperature dependences of dielectric permittivity of Rochelle salt near the upper transition point at different times of annealing in the paraelectric phase at $308 \mathrm{~K}(\mathrm{~min}): 1-0,2-5,3-20,4-60$. Inset: dependences of the maximal value of permittivity and maximum temperature on annealing time. 
parently caused by internal electrical bias fields whose magnitude is decreased by annealing.

Keeping the samples in the ferroelectric phase for a long term the internal bias fields caused by polar defects [25] participate in screening the spontaneous polarization and reflect the corresponding domain structure. The action of the internal bias field is analogous to the action of external field, that is, the temperature of the upper maximum of permittivity increases, and the maximum magnitude decreases. In the next section we shall estimate the magnitudes of internal bias fields in non-annealed and annealed samples.

\section{The effect of external electric field}

In figure 3 we show the measured temperature dependences of dielectric permittivity $\varepsilon_{11}$ of Rochelle salt crystals near the upper and lower transition points at different values of external d.c. electric field $E=E_{1}$ applied along the ferroelectric axis (conjugate to polarization). The insets contain the field dependences of the dielectric permittivity maxima $\varepsilon_{m}$ and their temperatures $\Delta T_{m}=T_{m}(E)-T_{m}(0)$. The data are obtained by cooling samples for the upper maximum and by heating for the lower one (from the corresponding paraelectric phase towards the ferroelectric phase). As expected, the external field, conjugate to polarization, decreases the $\varepsilon_{m}$ and shifts the maxima temperatures $\Delta T_{m}$ in a non-linear way. For the upper maximum $\Delta T_{m 2}>0$, whereas for the lower one $\Delta T_{m 1}<0$.
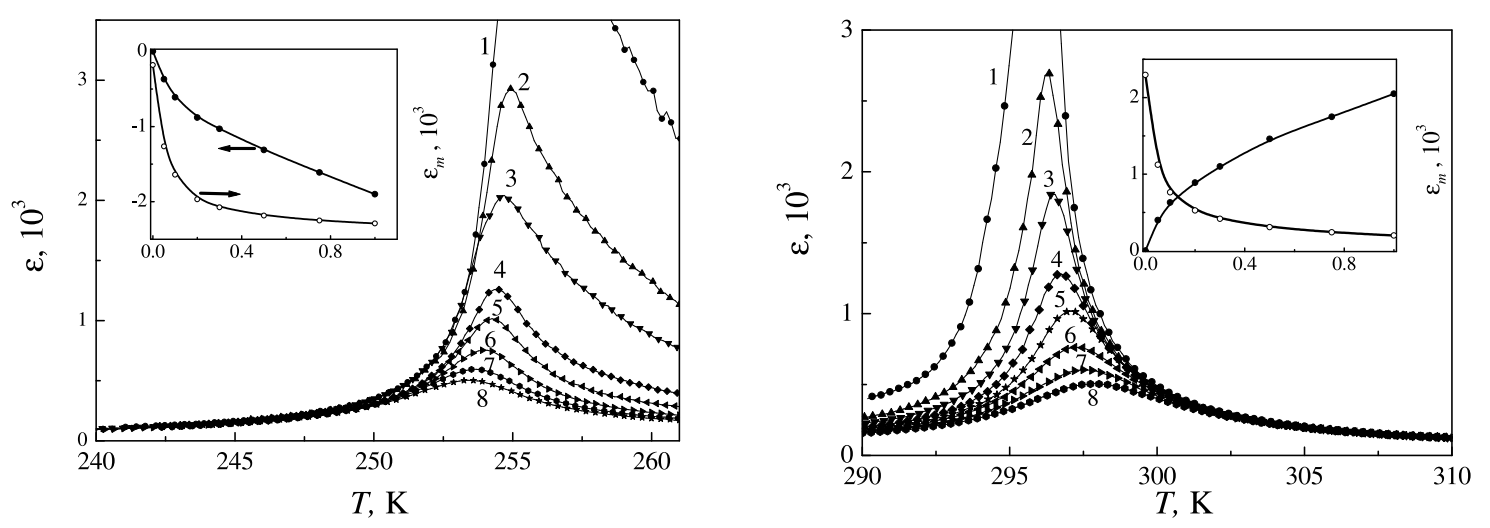

Figure 3. Temperature dependences of dielectric permittivity of Rochelle salt crystals near upper and lower transition points at different values of external electric field $E_{1}(\mathrm{kV} / \mathrm{cm}): 1-0,2-0.05,3-0.1,4-0.2,5-0.3,6-0.5,7-$ $0.75,8-1$. Lines are guide to the eyes.

These results are compared in figure 4 with literature data [26]. The field dependences of the permittivity maxima magnitudes $\varepsilon_{m}^{-1}(E)$ obtained in this work are the same for the two maxima (see figure 4) and well accord with the data of [26]. However, a perceptible disagreement is observed for the shift of permittivity maxima temperatures. Our data yield very close changes of $\left|\Delta T_{m}\right|$ with the field for the two 

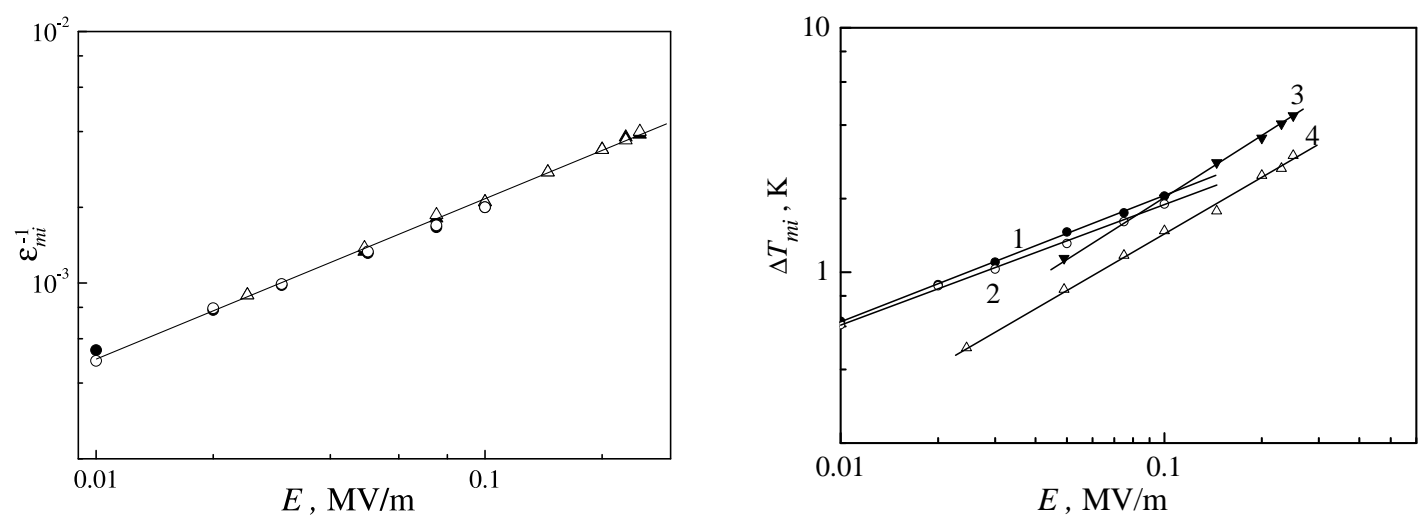

Figure 4. Field dependences of the permittivity maxima magnitudes (left) and temperature shifts (right). Upper maximum: line $1(\bullet)-$ this work, line $3(\mathbf{v})-$ [17]. Lower maximum - line $2(\circ)-$ this work, line $4(\Delta)-[17]$.

maxima. On the contrary, the field dependence of the upper maximum temperature obtained in [26] is much stronger than of the lower one.

For uniaxial ferroelectrics such as Rochelle salt, the phenomenological Landau expansion of thermodynamic potential can be presented as (elastic and piezoelectric contributions not considered)

$$
\Phi\left(P_{1}\right)=\Phi_{0}+\frac{\alpha}{2} P_{1}^{2}+\frac{\beta}{4} P_{1}^{4},
$$

where $P_{1}$ is the crystal polarization, $\alpha, \beta$ are the expansion coefficients. The electric field $E_{1}$ is applied along the axis of spontaneous polarization [100].

From (5.1) the following equations for polarization and inverse permittivity follow

$$
E_{1}=\left(\frac{\partial \Phi}{\partial P_{1}}\right)=\alpha P_{1}+\beta P_{1}^{3}, \quad \varepsilon_{11}^{-1}=\varepsilon_{0}\left(\frac{\partial E_{1}}{\partial P_{1}}\right)=\varepsilon_{0}\left(\alpha+3 \beta P_{1}^{2}\right)
$$

$\varepsilon_{0}$ is the dielectric permittivity of vacuum.

For Rochelle salt there are two possible ways to model the temperature dependence of the coefficient $\alpha$.

1. The expansion (5.1) is performed near each of the two transitions separately, assuming a linear temperature dependence $\alpha=\alpha_{\mathrm{T} 1}\left(T_{\mathrm{C} 1}-T\right)$ for the lower transition and $\alpha=\alpha_{\mathrm{T} 2}\left(T-T_{\mathrm{C} 2}\right)$ for the upper one. Then the field dependences of $\varepsilon_{m}\left(E_{1}\right)$ and $\Delta T_{m}\left(E_{1}\right)$ can be presented as [25]:

$$
\begin{gathered}
\varepsilon_{m}^{-1}=\frac{3}{2}(4 \beta)^{1 / 3} \varepsilon_{0} E_{1}^{2 / 3}=k_{1} E_{1}^{2 / 3} . \\
\left|\Delta T_{m i}\right|=\frac{3}{4} \frac{(4 \beta)^{1 / 3}}{\alpha_{\mathrm{T} i}} E_{1}^{2 / 3}=k_{2} E_{1}^{2 / 3}, \quad i=1,2 .
\end{gathered}
$$

2. Within the second approach, the coefficient $\alpha$ is chosen in the form

$$
\alpha=\alpha_{1}+\alpha_{2}\left(T-T_{0}\right)^{2},
$$


where $T_{0}=\frac{T_{\mathrm{C} 1}+T_{\mathrm{C} 2}}{2}$, and $T_{\mathrm{C} 1,2}=T_{0} \mp \sqrt{-\frac{\alpha_{1}}{\alpha_{2}}}$. Such a choice is supported by the fact that the phase transitions in Rochelle salt are close to a double critical point $[10,27]$, realized at partial substitution of potassium atoms with ammonia $\mathrm{NH}_{4}[11,28]$. In $[10,27]$ the temperature dependences of several physical characteristics of Rochelle salt were successfully described within the Landau approach with (5.5).

In this case, the field dependences of $\Delta T_{m}\left(E_{1}\right)$ are

$$
\Delta T_{m 1,2}= \pm A \mp \sqrt{\frac{3}{4} \frac{(4 \beta)^{1 / 3}}{\alpha_{\mathrm{T} 2}} E_{1}^{2 / 3}+A^{2}},
$$

where $A^{2}=-\alpha_{1} / \alpha_{2}$. The field dependence of $\varepsilon_{m}\left(E_{1}\right)$ in this case is the same as in the first approach and described by (5.3).

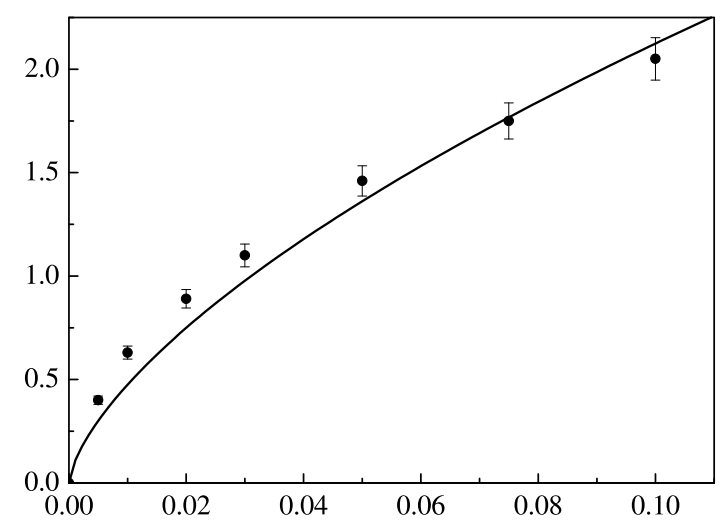

Figure 5. Field dependence of the upper permittivity maximum temperature shift. Line is calculated with (5.4). Symbols are experimental data of this work.

The experimental dependences of $\varepsilon_{m}^{-1}\left(E_{1}\right)$ of this work are well described by equation $(5.3)$ with $k_{1}=10.2 \cdot 10^{-7}(\mathrm{~m} / \mathrm{V})^{2 / 3}$ and $\beta=11.34 \cdot 10^{13} \mathrm{~V} \cdot \mathrm{m}^{5} / \mathrm{C}^{3}$. Fitting to the experimental data for $\Delta T_{m}\left(E_{1}\right)$ with equation (5.4), shown in figure 5 , yields the values of $k_{2}$ and $\alpha_{\mathrm{T} 1}, \alpha_{\mathrm{T} 2}$ for the upper and lower maxima:

for $T_{\mathrm{C} 1}: \quad k_{2}=9.9 \cdot 10^{-4} \mathrm{~K}(\mathrm{~m} / \mathrm{V})^{2 / 3}$ and $\alpha_{\mathrm{T} 1}=5.82 \cdot 10^{7} \mathrm{~V} \cdot \mathrm{m} \cdot(\mathrm{K} \cdot \mathrm{C})^{-1}$;

for $T_{\mathrm{C} 2}: \quad k_{2}=10.5 \cdot 10^{-4} \mathrm{~K}(\mathrm{~m} / \mathrm{V})^{2 / 3}$ and $\alpha_{\mathrm{T} 2}=5.49 \cdot 10^{7} \mathrm{~V} \cdot \mathrm{m} \cdot(\mathrm{K} \cdot \mathrm{C})^{-1}$.

The agreement with experiment for $\Delta T_{m}\left(E_{1}\right)$, obtained with formulas (5.6) is not any better than with (5.4). We found that

$$
\alpha_{1}=-5.82 \cdot 10^{8} \mathrm{~V} \cdot \mathrm{m} \cdot \mathrm{C}^{-1}, \quad \alpha_{2}=1.32 \cdot 10^{6} \mathrm{~V} \cdot \mathrm{m} \cdot \mathrm{C}^{-1} \cdot{ }^{-2} .
$$

The second approach is advantageous only at describing the physical characteristics of Rochelle salt in a sufficiently wide temperature range in paraelectric phases, where the non-linear temperature dependence of the inverse permittivity should be essential. For the description of the $\Delta T_{m}\left(E_{1}\right)$ dependences considered here, the nonlinearity of the coefficient $\alpha$ within a few Kelvins near the transition points does not play any significant role. 
Description of field dependences of dielectric permittivity of Rochelle salt within a modified Mitsui model with piezoeffect will be given elsewhere.

Using the above results, we can estimate the magnitude of internal bias fields existing in crystals without annealing as well as after $60 \mathrm{~min}$ of annealing. In the former and latter cases, the values of the permittivity at the upper transition point are about 3500 and 5100 (see figure 2). Therefore, using (5.3) and the found values of $k_{2}$, we get that at the upper Curie point $E_{\text {bias }}=0.055 \mathrm{kV} / \mathrm{cm}$ for a non-annealed sample and $E_{\text {bias }}=0.027 \mathrm{kV} / \mathrm{cm}$ for the sample annealed for $60 \mathrm{~min}$.

\section{External pressures}

\subsection{Uniaxial stresses}

The temperature dependences of dielectric permittivity $\varepsilon_{11}$ of Rochelle salt were measured at different values of mechanical stresses applied along the main crystallographic directions of unit cell: [100] $-\sigma_{1},[010]-\sigma_{2},[001]-\sigma_{3}$ and along [011] $\tilde{\sigma}_{4}$. In the reference system with axes along the main crystallographic directions, the stress $\tilde{\sigma}_{4}$ can be presented as

$$
\tilde{\sigma}_{4}=\sigma_{4}+\frac{1}{2}\left(\sigma_{2}+\sigma_{3}\right)
$$

where $\sigma_{4}$ is the shear strain, which for the Rochelle salt symmetry is the external field conjugate to the order parameter and acts similarly to the electric field $E_{1}$.
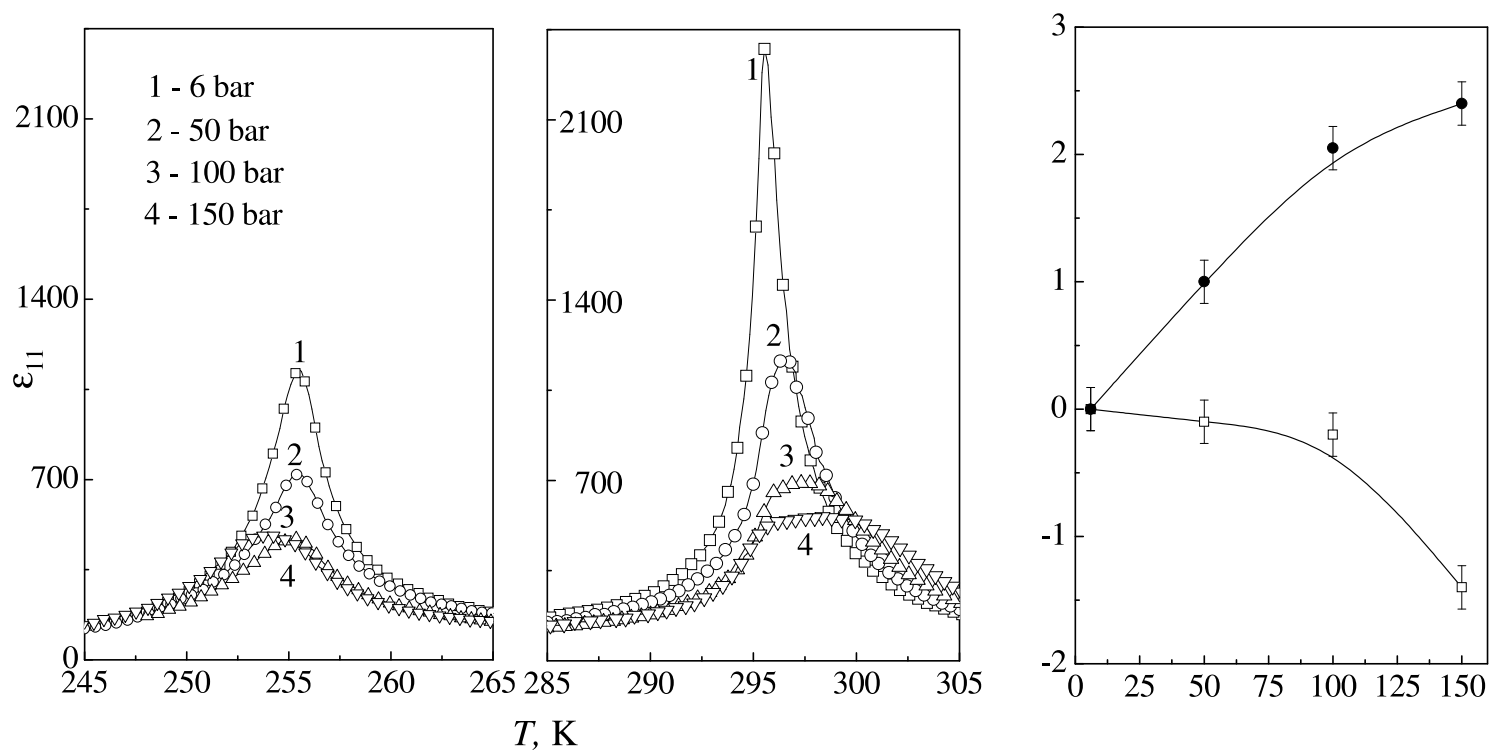

Figure 6. Temperature dependences of dielectric permittivities of Rochelle salt at different values of mechanical stress $\sigma_{1}$ and the stress dependences of permittivity maxima temperatures.

Figures 6-9 contain the obtained temperature dependences of dielectric permittivities at different values of uniaxial stresses and the corresponding stress dependences of the permittivity maxima temperatures. The data, as in the case of electric 
field study, were obtained at cooling for the upper maximum and at heating for the lower maximum (on going from the corresponding paraelectric phase towards the ferroelectric phase).
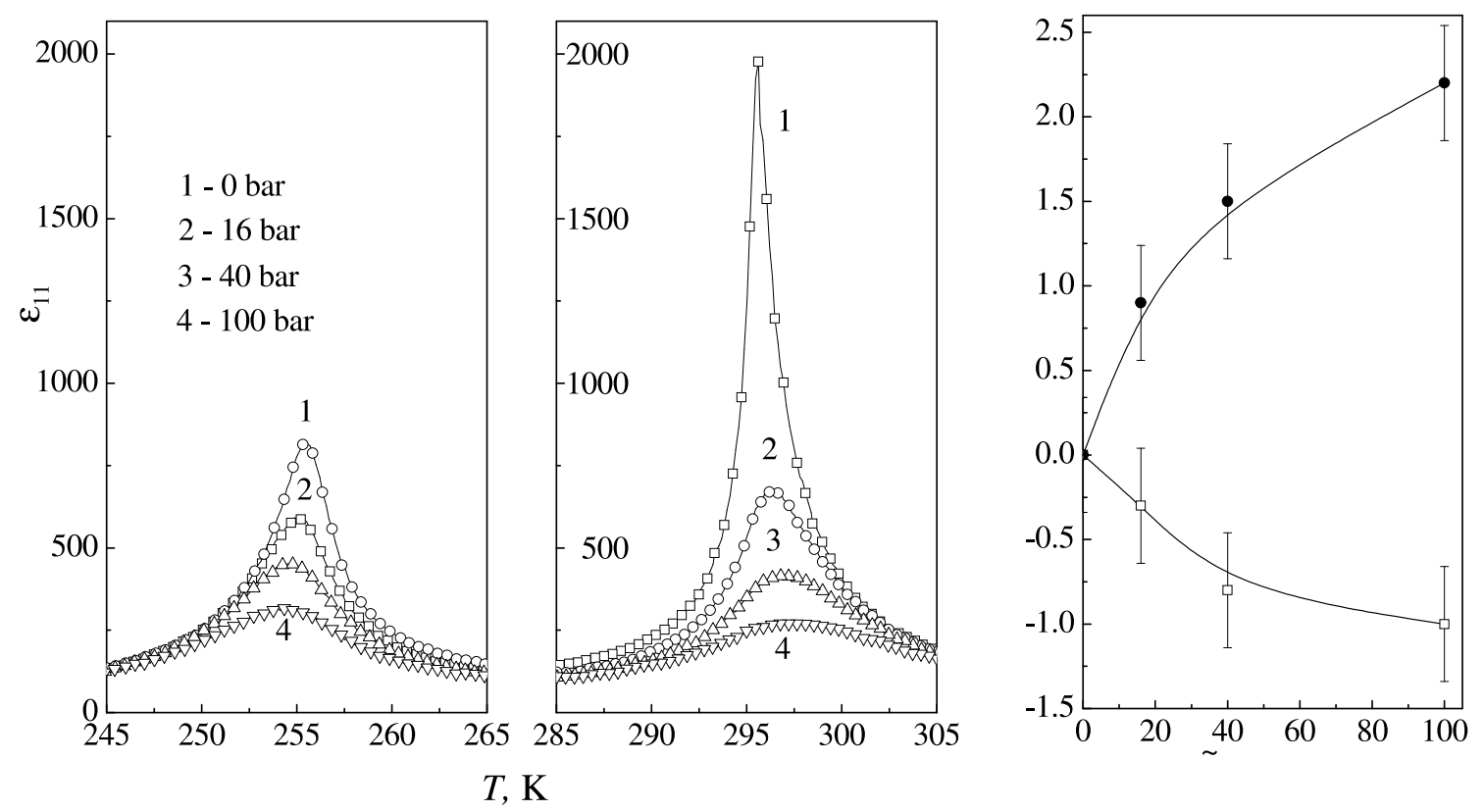

Figure 7. Same for the stress $\tilde{\sigma}_{4}$.
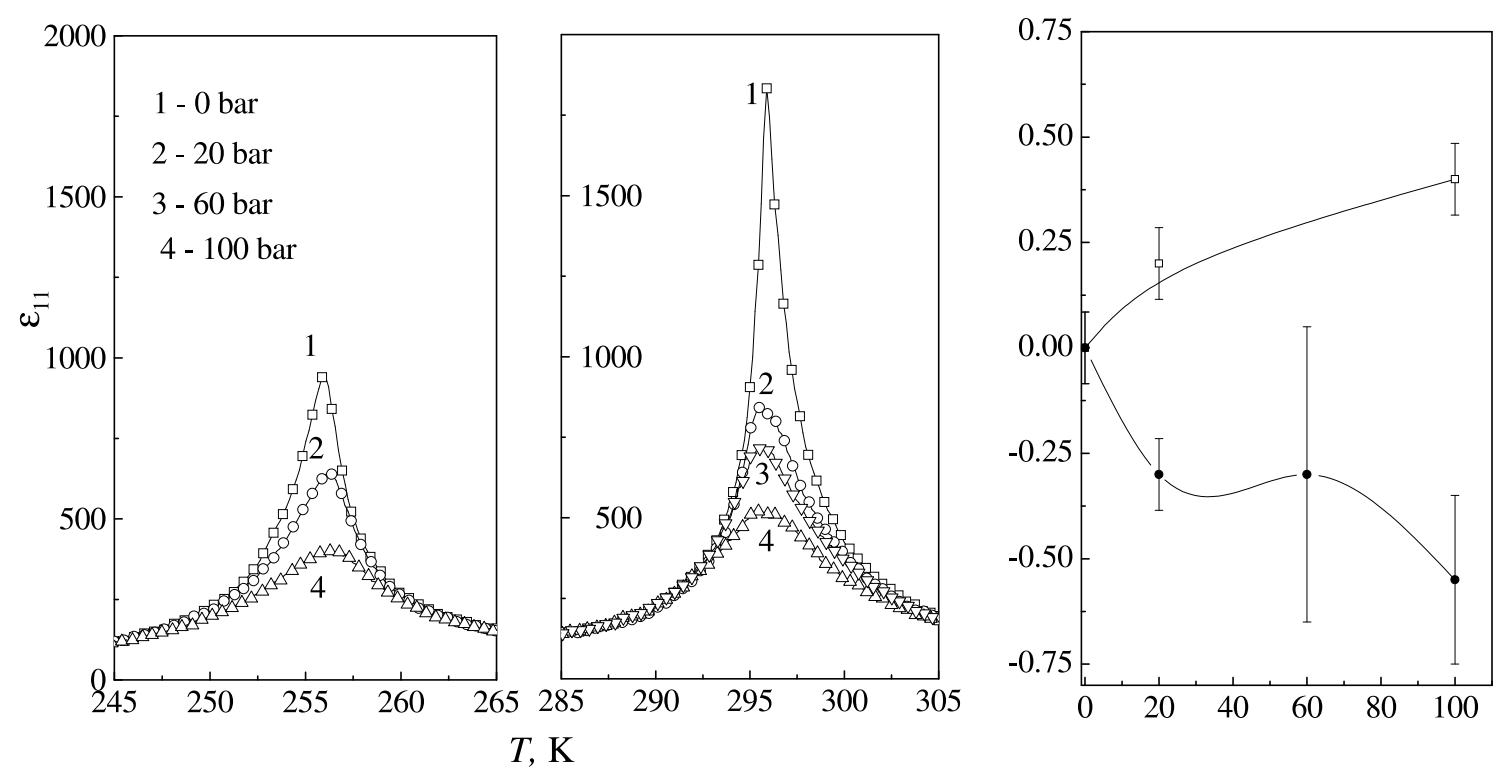

Figure 8. Same for the stress $\sigma_{2}$.

All the explored uniaxial stresses decrease the maximal values of dielectric permittivity and change their temperatures $T_{m 1}$ and $T_{m 2}$. The action of the stresses $\sigma_{1}$ and $\tilde{\sigma}_{4}$ on $T_{m 1}$ and $T_{m 2}$ is non-linear and similar to the action of the electric field 
$E_{1}: \mathrm{d} T_{m 1} / \mathrm{d} \sigma_{i}<0 ; \mathrm{d} T_{m 2} / \mathrm{d} \sigma_{i}>0(i=1$ and $\tilde{4})$. The change of the upper maximum temperature with the stress $\tilde{\sigma}_{4}$ is much larger than of the lower maximum. Let us remind that the changes of the maxima temperatures with the electric field $E_{1}$ obtained in this work are almost the same for the two maxima (see figures 4, 5).
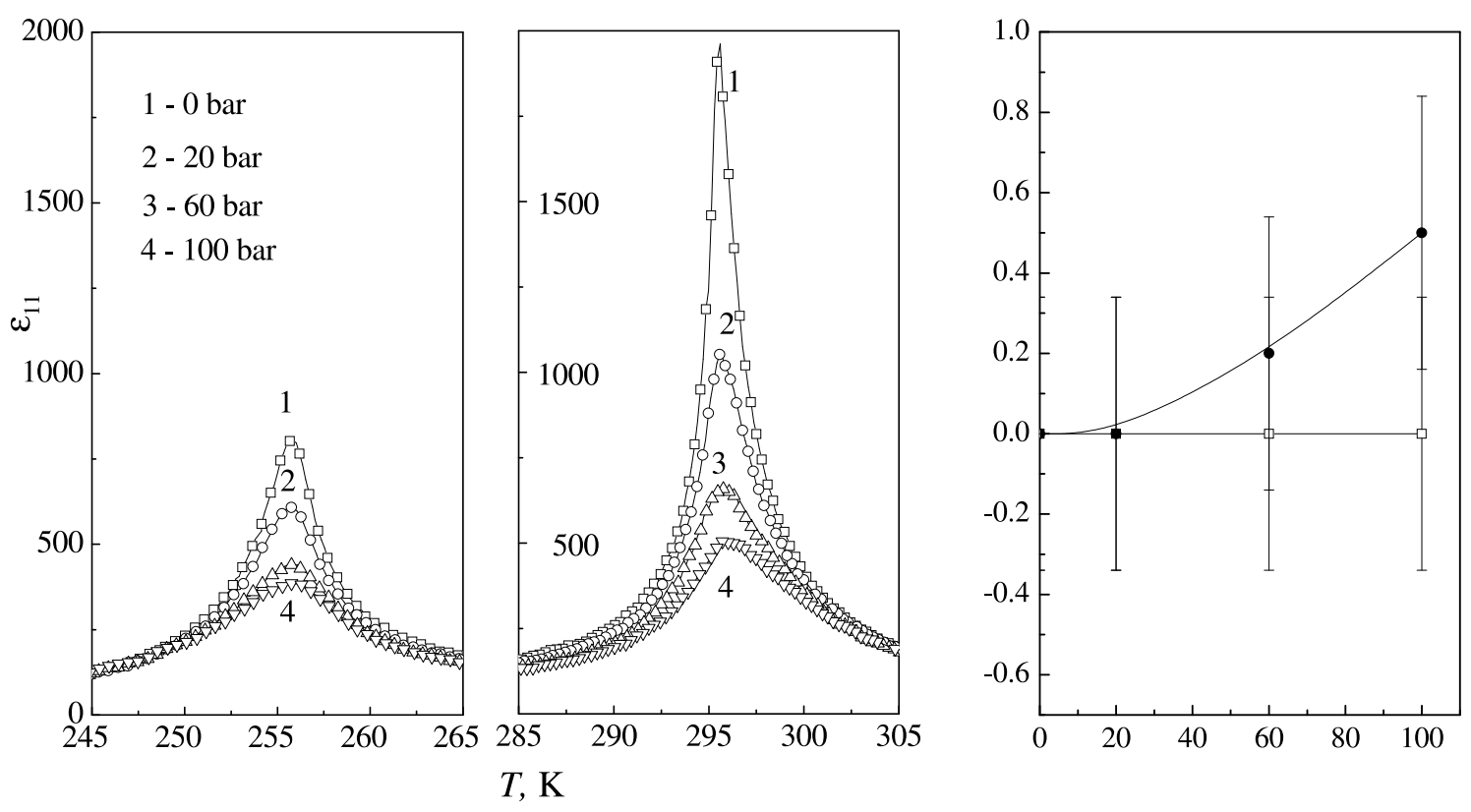

Figure 9. Same for the stress $\sigma_{3}$.

In section 6.3 we shall compare our results with the corresponding literature data [13] obtained from the thermoelastic effect studies and with the data of the phenomenological analysis. As we shall see, our data qualitatively agree with the literature, except for the case of stress $\sigma_{3}$. However, the quantitative agreement is rather poor, our data for $\left|\Delta T_{\mathrm{Ci}}\right|$ being a few times smaller.

\subsection{Hydrostatic pressure}

Figure 10 contains the temperature dependences of the dielectric permittivity of Rochelle salt at different hydrostatic pressures. In contrast to electric field or uniaxial stresses, the hydrostatic pressure increases the both transition temperatures (see the inset with the $p, T$-diagram). The pressure coefficients of transition temperatures are $\mathrm{d} T_{\mathrm{C} 1} / \mathrm{d} p=3.54 \mathrm{~K} / \mathrm{kbar}$ and $\mathrm{d} T_{\mathrm{C} 2} / \mathrm{d} p=10.92 \mathrm{~K} / \mathrm{kbar}$, in perfect agreement with the data of $[7,8]$. On increasing the hydrostatic pressure, the value of $\varepsilon_{m}$ at the lower transition point $T_{\mathrm{C} 1}$ monotonously decreases but remains unchanged at $T_{\mathrm{C} 2}$. 


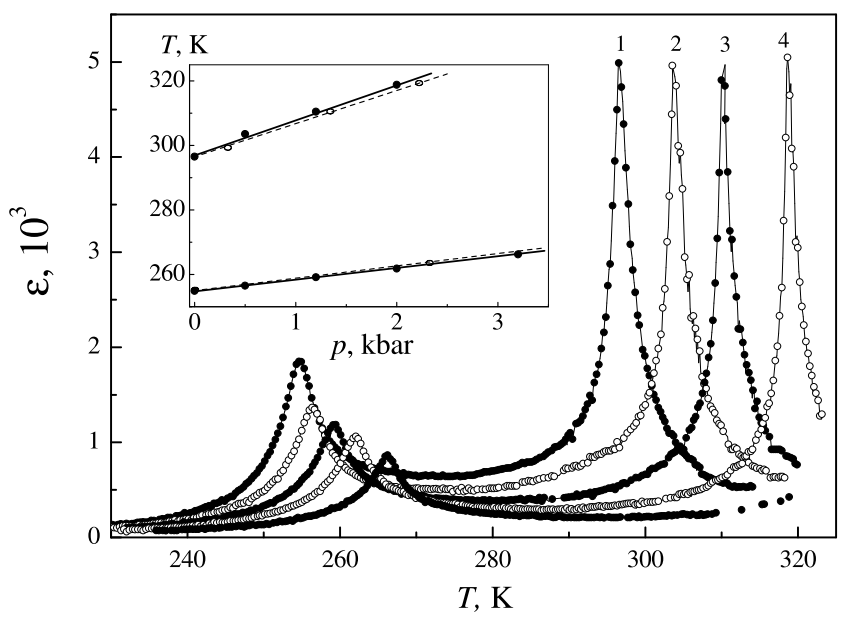

Figure 10. Temperature dependence of the dielectric permittivity of Rochelle salt at different values of hydrostatic pressure $p, \mathrm{MPa}: 1-0 ; 2-50 ; 3-120 ; 4-200$; 5 - 320. (Inset: the $p, T$ phase diagram. Dashed lines and $\circ$ - data of [7].)

\subsection{Phenomenological description of pressure effects}

For phenomenological description of external pressure effects on the phase transitions in Rochelle salt, let us modify the expansion (5.1) in the following way

$$
\Phi\left(P_{1}, \sigma_{i}\right)=\Phi_{0}+\frac{\alpha}{2} P_{1}^{2}+\frac{\beta}{4} P_{1}^{4}+\sum_{i=1}^{3} q_{i 1} \sigma_{i} P_{1}^{2}+g_{14} P_{1} \sigma_{4}-\frac{1}{2} \sum_{i j=1}^{4} s_{i j}^{P} \sigma_{i} \sigma_{j} .
$$

For the sake of simplicity we changed here the signs of the stresses $\sigma_{i}$ as compared to the standard notations, so that the uniaxial compression stresses are positive, and for the hydrostatic pressure we have $p=\sigma_{1}=\sigma_{2}=\sigma_{3}$. In standard notations $-p=\sigma_{1}=\sigma_{2}=\sigma_{3}$ and the values of the compression stress are negative.

The quantities $q_{i 1}$ are the electrostriction coefficients; $s_{i j}^{P}$ are the elastic compliances at constant polarization. Let us note that $s_{i j}^{P}$ for $i=1,2,3$ and $s_{44}^{P}$ are practically temperature independent, whereas $s_{i 4}^{P} \sim P_{1}$, that is, they are different from zero only in the ferroelectric phase or in the presence of electric field (possibly internal bias field $E_{\text {bias }}$ due to polar defects) or stress $\sigma_{4}$.

From (6.2) the equations for polarization and lattice strains follow

$$
\begin{aligned}
& E_{1}=g_{14} \sigma_{4}+\left(\alpha+2 \sum_{i=1}^{3} q_{i 1} \sigma_{i}\right) P_{1}+\beta P_{1}^{3}-\sum_{i=1}^{3} \frac{s_{i 4}^{P}}{P_{1}} \sigma_{i} \sigma_{4}, \\
& u_{i}=\frac{\partial \Phi}{\partial \sigma_{i}}=-\sum_{j=1}^{4} s_{i j}^{P} \sigma_{j}+q_{i 1} P_{1}^{2}, \quad i=1,2,3, \\
& u_{4}=g_{14} P_{1}-\sum_{j=1}^{4} s_{i j}^{P} \sigma_{j} .
\end{aligned}
$$

Assuming a linear dependence of the coefficient $\alpha=\alpha_{\mathrm{T} 1}\left(T_{\mathrm{C} 1}-T\right)$ for the lower transition and $\alpha=\alpha_{\mathrm{T} 2}\left(T-T_{\mathrm{C} 2}\right)$ for the upper one, we get for the transition temperature 
shift and the inverse values of permittivity of a free crystal (at constant stress)

$$
\begin{aligned}
\Delta T_{C 1,2} & = \pm \frac{2}{\alpha_{\mathrm{T} 1,2}} \sum_{i=1}^{3} q_{i 1} \sigma_{i} \mp k_{1}\left(E_{\mathrm{bias}}-g_{14} \sigma_{4}\right)^{2 / 3} \\
\varepsilon_{m 1,2}^{-1} & =k_{2}\left(E_{\mathrm{bias}}-g_{14} \sigma_{4}+\sum_{i=1}^{3} \frac{s_{i 4}^{P}}{P_{1}} \sigma_{i} \sigma_{4}\right)^{2 / 3}
\end{aligned}
$$

Experimental data for $g_{14}$ are rather dispersive (see the systematization in [15]). We used here the theoretical data for $g_{14}$ of [15], that agree overall with the experiment. The electrostriction coefficients have been determined in [29]. We adjust here their values, in order to get a good fit to the hydrostatic pressure dependences of transition temperatures. The values of $q_{i 1}, g_{14}$ at lower and upper transition points are given in table 2 .

Table 2. The data for $q_{i 1}$ (in $\left.\mathrm{m}^{4} / \mathrm{C}^{2}\right)$ and $g_{14}\left(\right.$ in $\mathrm{m}^{2} / \mathrm{C}$ ).

\begin{tabular}{c|ccc|c}
\hline & $q_{11}$ & $q_{21}$ & $q_{31}$ & $g_{14}$ \\
\hline$T_{\mathrm{C} 1}$ & -7.5 & 4 & 4.5 & 0.174 \\
$T_{\mathrm{C} 2}$ & -10 & 4.3 & 2.5 & 0.195 \\
\hline
\end{tabular}

First we consider the case of a perfect crystal $\left(E_{\text {bias }}=0\right)$. The calculated shifts of transition temperatures (permittivity maxima temperatures) with uniaxial and hydrostatic pressures are presented in table 3 . As one can see, a very good agreement is obtained with the hydrostatic pressure data, as well as the data of [13] for the uniaxial stresses. The agreement with the data for $\tilde{\sigma}_{4}$ is completely unsatisfactory. Here we used an assumption that, according to (6.1), $\tilde{\sigma}_{4}=100$ bar corresponds to a sum of $\sigma_{4}=50$ bar, $\sigma_{2}=50$ bar, $\sigma_{3}=50$ bar. Our data for the uniaxial stresses $\sigma_{1}, \sigma_{2}, \sigma_{3}$ are also in poor agreement with the phenomenology and with the literature data; however, the calculations agree with [13] fairly well.

Table 3. Shifts of the transition temperatures with uniaxial stresses (per 100 bar)

\begin{tabular}{|c|c|c|c|c|c|c|c|c|c|c|c|c|c|}
\hline & \multicolumn{3}{|c|}{$\sigma_{1}$} & \multicolumn{3}{|c|}{$\sigma_{2}$} & \multicolumn{3}{|c|}{$\sigma_{3}$} & \multicolumn{2}{|c|}{$\tilde{\sigma}_{4}$} & \multicolumn{2}{|c|}{ hydrostatic } \\
\hline & exp. & [13] & calc. & exp. & [13] & calc. & exp. & [13] & calc. & exp. & calc. & exp. & calc. \\
\hline$T_{\mathrm{C} 1}$ & -1.2 & -2.9 & -2.73 & 0.4 & 1.5 & 1.46 & 0 & 1.7 & 1.63 & -1.0 & -8.8 & 3.43 & 3.64 \\
\hline$T_{\mathrm{C} 2}$ & 2.0 & 3.5 & 3.44 & -0.6 & -1.6 & -1.48 & 0.5 & -0.8 & -0.86 & 2.2 & 9.1 & 10.92 & 10.99 \\
\hline
\end{tabular}
and with hydrostatic pressure (per $1 \mathrm{kbar}$ ).

It seems likely that the disagreement between the experimental data of this work and of [13] should be attributed to the effect of sample defects. We recalculated the shifts of the maxima temperature with uniaxial pressure taking into account the role of internal bias field, determining them from (6.7). A much better agreement was 
obtained for the stresses $\sigma_{2}$ and $\sigma_{3}:\left|\Delta T_{\mathrm{Ci}}\right|$ decrease by several times with increasing $E_{\text {bias }}=0$. However, for the stress $\sigma_{1}$, the presence of the bias field has further enhanced the theoretical values $\left|\Delta T_{\mathrm{Ci}}\right|$, only worsening the agreement with the experimental data of this work. At the moment, we have no satisfactory explanation of the disagreement between our experimental data and the data of [13] and of the calculations, especially in view of the fact that for hydrostatic pressure a complete coincidence with the literature data and with phenomenology has been obtained.

The origin of a strong decrease of permittivity maxima magnitude with diagonal stresses $\sigma_{i}, i=1,2,3$ is not quite clear either. As it follows from (6.7), such a decrease can be accounted for by the increase of the internal bias field $E_{\text {bias }}$ or of the coefficient $k_{2} \sim \beta^{1 / 3}$. Such an increase of $k_{2}$ can be obtained if we take into account the terms of the fourth order of the $\sum_{i=1}^{3} q_{i 1}^{(4)} \sigma_{i} P_{1}^{4}$ type in the expansion (6.2). Effectively it would lead to renormalization of the coefficient $\beta \rightarrow \beta+4 \sum_{i=1}^{3} q_{i 1}^{(4)} \sigma_{i}$.

\section{Concluding remarks}

- Strong dependence of dielectric permittivity of Rochelle salt on humidity of the storage air is shown. We believe that the dispersion of experimental data of different literature sources can be caused by the uncontrolled water content during and prior to the measurements.

- The dependence of the permittivity value at the transition points on the duration of thermal annealing in high-temperature paraelectric phase indicates the existence of internal electric bias fields in the crystals due to the point polar defects.

- The effect of external electric field, uniaxial stresses, and hydrostatic pressure on the dielectric permittivity has been studied. The results are compared with the available literature data. The analysis of the obtained results is performed within the phenomenological Landau approach. Possible reasons for discrepancies in the data are discussed.

\section{Acknowledgement}

The authors acknowledge support of Fundamental Researches State Fund of Ukraine, project No. 02.07/00310.

\section{References}

1. Stasyuk I.V., Levitskii I.V., Moina A.P., Phys. Rev. B., 1999, 59, 8530.

2. Stasyuk I.V. Levitskii R.R., Zachek I.R., Moina A.P., Phys. Rev B., 2000, 62, 6198.

3. Stasyuk I.V., Levitskii R.R., Zachek I.R., Duda A.S., Condens. Matter Phys., 2001, 4, 553 .

4. Levitskii R.R., Slivka A.G., Moina A.P. et al, Journ. Phys. Studies., 2002, 6, No. 2, 197. 
5. Stasyuk I.V., Levitskii R.R. et al, Journ. Phys. Studies, 1999, 3, 502.

6. Stasyuk I.V., Levitskii R.R., Moina A.P., Velychko O.V., Ferroelectrics, 2003, 288, 133.

7. Bancroft D., Phys. Rev., 1938, 53, 587.

8. Samara G.A., J. Chem. Solids, 1965, 26, 121.

9. Samara G.A., J. Chem. Solids, 1969, 29, 870.

10. Serdobolskaya O.Yu., Sol. Stat. Phys., 1996 38, 1529.

11. Gesi K., Ozawa K., J. Phys. Soc. Japan, 1992, 48, 2003.

12. Ismailzade I.H., Samedov O.A., Alekberov A.I., Phys. Stat. Sol. A, 1986, 94, K17.

13. Imai K., Journ. Phys. Soc. Japan, 1975, 39, 868.

14. Levitskii R.R., Zachek I.R., Moina A.P., Verkholyak T.M., J. Phys. Studies, 2003, 7, 106.

15. Levitskii R.R., Zachek I.R., Verkholyak T.M., Moina A.P., Phys. Rev. B., 2003, 67, 174112.

16. Mason W.P., Piezoelectric Crystals and Their Application to Ultrasonics. Van Nostrand, New York, 1950.

17. Valasek J., Phys. Rev., 1922, 20, 639.

18. Unruh H.-G., Sailer E.Z., Phys. A, At. Nuclei (West Germany), 1969, 224, No. 1-3, 45-64.

19. Hablützel J., Helv. Phys. Acta, 1939, 12, 489.

20. Taylor W., Lockwood D.J., Labbe H.J., J. Phys. C.: Solid State Phys., 1984, 17, 3685.

21. Mueller H., Phys. Rev., 1935, 47, 175.

22. Mason W.P., Phys. Rev., 1939, 55, 775.

23. Petrov V.M., Kristallografiya (Sov. Phys. - Crystallogr.), 1962, 7, 403.

24. Yurin V.A., Bull. Ac. Sci USSR, Phys. Ser., 1965, 29, 2001.

25. Lines M., Glass A. Principles and application of ferroelectrics and related materials. Clarendon Press, Oxford, 1977.

26. Akishige Y., Kamishina Y., J. Phys. Soc. Japan, 1992, 61, 4589.

27. Kryukova E.B., Sobyanin A.A., Bull. Ac. Sci USSR, Phys. Ser. , 1987, 512090.

28. Makita Y., Takagi Y., J. Phys. Soc. Japan, 1958, 13, 367.

29. Schmidt G., Z. Phys., 1961, 164, 579. 


\title{
Вплив зовнішніх факторів на діелектричну проникність сегнетової солі: вологість, відпал, тиски, електричне поле
}

\author{
О.Г.Сливка ${ }^{1}$, В.М.Кедюлич ${ }^{1}$, Р.Р.Левицький ${ }^{2}$, \\ А.П.Моїна ${ }^{2}$, М.О.Романюк ${ }^{3}$, Г.М.Гуйван ${ }^{1}$ \\ 1 Ужгородський національний університет, \\ Ужгород 88000, вул. Волошина, 54 \\ 2 Інститут фізики конденсованих систем, \\ Львів 79011, вул. Свєнціцького, 1 \\ 3 Львівський національний університет ім. І.Франка, \\ Львів 79005, вул. Кирила і Мефодія, 8
}

Отримано 18 травня 2005 р.

Робота містить результати експериментальних досліджень впливу зовнішніх факторів таких, як, висушування/зволоження, термічний відпал, електричне поле, одновісні тиски, гідростатичний тиск, на діелектричну проникність кристалів сегнетової солі. Отримані результати порівнюються з відомим літературними даними та аналізуються в рамках феноменологічного підходу. Показано, що умови зберігання та внутрішні полярні точкові дефекти суттєво впливають на діелектричну проникність кристалів.

Ключові слова: сегнетова сіль, тиск, електричне поле, дефекти, відпал, вологість

PACS: $77.80 . B h, 77.22 . C h$ 